\title{
Ipatasertib plus paclitaxel for PIK3CA/AKT1/PTEN-altered hormone receptor-positive HER2-negative advanced breast cancer: primary results from cohort B of the IPATunity 130 randomized phase 3 trial
}

\author{
Nicholas Turner ${ }^{1,2}$ (D) $\cdot$ Rebecca A. Dent ${ }^{3}$. Joyce O'Shaughnessy ${ }^{4} \cdot$ Sung-Bae Kim ${ }^{5} \cdot$ Steven J. Isakoff $^{6} \cdot$ Carlos Barrios $^{7}$. \\ Shigehira Saji ${ }^{8} \cdot$ Igor Bondarenko $^{9} \cdot$ Zbigniew Nowecki $^{10} \cdot$ Qinshu Lian $^{11} \cdot$ Sarah-Jayne Reilly $^{12} \cdot$ Heather Hinton $^{13}$. \\ Matthew J. Wongchenko ${ }^{14} \cdot$ Bruno Kovic $^{15} \cdot$ Aruna Mani $^{16} \cdot$ Mafalda Oliveira $^{17}$
}

Received: 22 April 2021 / Accepted: 12 November 2021 / Published online: 3 December 2021

(c) The Author(s) 2021

\begin{abstract}
Purpose PI3K/AKT pathway alterations are frequent in hormone receptor-positive (HR+) breast cancers. IPATunity130 Cohort B investigated ipatasertib-paclitaxel in PI3K pathway-mutant HR + unresectable locally advanced/metastatic breast cancer $(\mathrm{aBC})$.

Methods Cohort B of the randomized, double-blind, placebo-controlled, phase 3 IPATunity130 trial enrolled patients with HR+HER2-negative PIK3CA/AKT1/PTEN-altered measurable aBC who were considered inappropriate for endocrine-based therapy (demonstrated insensitivity to endocrine therapy or visceral crisis) and were candidates for taxane monotherapy. Patients with prior chemotherapy for aBC or relapse $<1$ year since (neo)adjuvant chemotherapy were ineligible. Patients were randomized 2:1 to ipatasertib (400 mg, days 1-21) or placebo, plus paclitaxel $\left(80 \mathrm{mg} / \mathrm{m}^{2}\right.$, days 1 , 8,15$)$, every 28 days until disease progression or unacceptable toxicity. The primary endpoint was investigator-assessed progression-free survival (PFS). Results Overall, 146 patients were randomized to ipatasertib-paclitaxel and 76 to placebo-paclitaxel. In both arms, median investigator-assessed PFS was 9.3 months (hazard ratio, 1.00, 95\% CI 0.71-1.40) and the objective response rate was $47 \%$. Median paclitaxel duration was 6.9 versus 8.8 months in the ipatasertib-paclitaxel versus placebo-paclitaxel arms, respectively; median ipatasertib/placebo duration was 8.0 versus 9.1 months, respectively. The most common grade $\geq 3$ adverse events were diarrhea (12\% with ipatasertib-paclitaxel vs $1 \%$ with placebo-paclitaxel), neutrophil count decreased (9\% vs $7 \%$ ), neutropenia ( $8 \%$ vs 9\%), peripheral neuropathy (7\% vs 3\%), peripheral sensory neuropathy (3\% vs 5\%) and hypertension $(1 \%$ vs $5 \%)$.
\end{abstract}

Conclusion Adding ipatasertib to paclitaxel did not improve efficacy in PIK3CA/AKT1/PTEN-altered HR+ HER2-negative $\mathrm{aBC}$. The ipatasertib-paclitaxel safety profile was consistent with each agent's known adverse effects.

Trial registration NCT03337724.

Keywords PI3K/AKT $\cdot$ Hormone receptor positive $\cdot$ HER2 negative $\cdot$ Ipatasertib · First-line

$\begin{array}{ll}\text { Abbreviations } \\ \text { aBC } & \text { Advanced or metastatic breast cancer } \\ \text { AE } & \text { Adverse event } \\ \text { CDK } & \text { Cyclin-dependent kinase } \\ \text { CI } & \text { Confidence interval } \\ \text { CTA } & \text { Clinical Trial Assay } \\ \text { EORTC } & \text { European Organisation for Research and } \\ & \text { Treatment of Cancer }\end{array}$

Nicholas Turner

Nick.Turner@icr.ac.uk

Extended author information available on the last page of the article
GHS/QoL Global Health Status/Quality of Life

HER- HER2-negative

$\mathrm{HR}+\quad$ Hormone receptor-positive

IRC Independent review committee

ITT Intent-to-treat

mTOR Mammalian target of rapamycin

OS Overall survival

PFS Progression-free survival

PI3K Phosphoinositide 3-kinase

PROs Patient-reported outcomes

QLQ-C30 Quality of Life Questionnaire Core 30

RECIST Response Evaluation Criteria in Solid Tumors

TNBC Triple-negative breast cancer 


\section{Introduction}

The phosphoinositide 3-kinase (PI3K)/AKT pathway is frequently upregulated in cancer $[1,2]$. Activation of AKT, the central node of the PI3K/AKT pathway, promotes cell survival, proliferation, metabolism and growth [1,3], and is implicated in resistance to endocrine therapy [4]. PIK3CA/AKTI/PTEN alterations are frequently observed in breast cancer, including approximately $50 \%$ of patients with hormone receptor-positive $(\mathrm{HR}+)$ breast cancers, and contribute towards a negative prognosis and resistance to endocrine therapies [5-9].

Ipatasertib is a highly selective oral ATP-competitive smallmolecule inhibitor of all three AKT isoforms [10]. Ipatasertib is being developed for the treatment of cancers in which PI3K/ AKT pathway activation may be relevant for tumor growth or therapeutic resistance, and has demonstrated PI3K/AKT pathway inhibition in preclinical studies [10-12]. PTEN protein loss and PTEN or PIK3CA genetic alterations appeared to be associated with enhanced sensitivity to single-agent ipatasertib in cell lines and preclinical models $[10,13]$. In a phase $1 \mathrm{~b}$ study, the combination of ipatasertib and paclitaxel was well tolerated and showed radiographic responses in patients with advanced/metastatic breast cancer, including HR+ disease [14]. In the randomized, phase 2 LOTUS trial, the addition of ipatasertib to paclitaxel improved progression-free survival (PFS) compared with paclitaxel alone in metastatic triple-negative breast cancer (TNBC), especially in patients whose tumors harbored alterations in PIK3CA, AKT1 and/or PTEN [15].

The phase 3 IPATunity 130 trial included two independent randomized cohorts (Cohort A in TNBC and Cohort B in HR+HER2-negative [HER2-] unresectable locally advanced or metastatic breast cancer $[\mathrm{aBC}]$ ) evaluating ipatasertib plus paclitaxel combination therapy and a third single-arm signalseeking cohort in patients with TNBC whose tumors did not have PIK3CA/AKTI/PTEN alterations (Cohort C) evaluating a triplet combination of ipatasertib, paclitaxel and atezolizumab. The two randomized cohorts are powered independently and designed to be analyzed separately. Here we report results from Cohort $\mathrm{B}$, which evaluated ipatasertib in combination with paclitaxel for HR+HER2- PIK3CA/AKTI/PTEN-altered aBC.

\section{Patients and methods}

\section{Study design and participants}

In Cohort B of the IPATunity130 (NCT03337724) randomized, double-blind, placebo-controlled, phase 3 trial, eligible patients had to have $\mathrm{HR}+(\geq 1 \%$ staining $)$ HER2- PIK3CA/AKT1/PTEN-altered measurable aBC according to Response Evaluation Criteria in Solid Tumors
(RECIST; version 1.1). Tumor PIK3CA/AKT1/PTEN alteration status (i.e., activating alterations in $P I K 3 C A$ and/or $A K T 1$, and/or inactivating alterations in PTEN, described in detail in Supplementary Table S1) was determined from the most recently available tumor tissue sample using the Foundation Medicine Inc (Cambridge, MA) next-generation sequencing Clinical Trial Assay (CTA). In addition, patients had to be inappropriate for endocrine-based therapy (i.e., demonstrated insensitivity to endocrine therapy or visceral crisis), a candidate for taxane monotherapy and have Eastern Cooperative Oncology Group performance status 0 or 1 . Patients who had previously received chemotherapy for $\mathrm{aBC}$ or whose diagnosis of $\mathrm{aBC}$ was $<1$ year since their last (neo)adjuvant chemotherapy were ineligible, as were patients with a history of or known presence of brain or spinal cord metastases. Prior cyclin-dependent kinase (CDK)4/6 inhibitors and PI3K/mammalian target of rapamycin (mTOR) inhibitors were permitted.

\section{Procedures}

Patients were randomized in a 2:1 ratio by investigators using an interactive web-response system to receive either oral ipatasertib (400 mg daily on days $1-21$ ) plus intravenous paclitaxel $\left(80 \mathrm{mg} / \mathrm{m}^{2}\right.$ on days 1,8 and 15$)$ of a 28 -day cycle, or placebo plus the same paclitaxel regimen. Randomization was stratified by three criteria: (neo)adjuvant chemotherapy (yes vs no), prior $\mathrm{PI} 3 \mathrm{~K} / \mathrm{mTOR}$ inhibitor (yes vs no) and region (Asia-Pacific vs Europe vs North America vs rest of the world). To improve the management of diarrhea (commonly associated with ipatasertib and/or paclitaxel therapy), antidiarrheal prophylaxis (loperamide) was mandated for the first cycle for all patients, where permitted locally. Treatment was continued until disease progression (RECIST; version 1.1), unacceptable toxicity or patient withdrawal. Patients discontinuing paclitaxel or ipatasertib/placebo because of toxicity could continue on single-agent treatment. Crossover from placebo to ipatasertib was not permitted.

Tumors were assessed every 8 weeks by the investigators according to RECIST (version 1.1). After discontinuing treatment, patients were followed up every 3 months for survival and subsequent anticancer therapies. Patientreported outcomes (PROs) were assessed using selected scales of the European Organisation for Research and Treatment of Cancer Quality of Life Questionnaire Core 30 (EORTC QLQ-C30), administered at baseline, at day 1 of each subsequent cycle, and at the treatment discontinuation visit. Adverse events (AEs) were assessed and graded according to Common Terminology Criteria for Adverse Events (version 4.0). 


\section{Endpoints}

The primary objective was to assess the efficacy of the ipatasertib plus paclitaxel combination as determined by investigator-assessed PFS. PFS was defined as the interval between randomization and the first occurrence of disease progression, as determined by the investigator according to RECIST (version 1.1), or death from any cause, whichever occurred first. A sensitivity analysis of PFS according to independent review committee (IRC) assessment was performed in a similar manner.

Overall survival (OS; defined as the interval between randomization and death from any cause) was the key secondary endpoint. Other secondary endpoints included confirmed objective response rate (investigator assessed per RECIST [version 1.1]), duration of response in responding patients, clinical benefit rate (complete or partial response, or stable disease sustained for $\geq 24$ weeks) in patients with measurable disease at baseline, PROs, and safety.

\section{Statistical analysis}

The planned sample size was 201 patients. For the primary analysis, $150 \mathrm{PFS}$ events in the intent-to-treat (ITT) population were required to detect a hazard ratio of 0.62 with $80 \%$ power at a two-sided significance level of 5\%. This corresponds to an increase in median PFS from 8.5 months in the control arm to 13.8 months in the ipatasertib-containing arm. If investigator-assessed PFS in the ITT population was significant at 5\%, OS was to be tested hierarchically at the same significance level.

Efficacy analyses were based on all randomly assigned patients (ITT population) according to the treatment arm to which patients were allocated. PRO analyses of Global Health Status/Quality of Life (GHS/QoL) were performed on randomized patients who had a baseline and at least one post-baseline PRO assessment (PRO-evaluable population); PRO analyses of time to $\geq 11$-point confirmed deterioration in pain [16] were performed on the ITT population. Safety analyses were based on all patients who received at least one dose of ipatasertib, placebo or paclitaxel; patients were analyzed based on the treatment actually received.

\section{Results}

\section{Patient population}

Between January 6, 2018 and March 29, 2019, 782 patients were screened for the trial, of whom 560 were considered screen failures, most commonly because of absence of PIK3CA/AKTI/PTEN alteration $(n=303)$. Ultimately, 222 patients were randomized: 146 to ipatasertib plus paclitaxel and 76 to placebo plus paclitaxel. Two patients in the ipatasertib plus paclitaxel arm were included in the efficacy analyses despite the most recent hormone receptor status identifying tumors as TNBC. Two patients (one in each arm) received no treatment and were therefore excluded from the safety analysis population (Fig. 1). The majority of samples for determination of PIK3CA/AKT1/ PTEN status were from primary tumor tissue (143 [64\%] primary, 66 [30\%] metastatic, 10 [5\%] unknown, three [1\%] enrolled based on local testing with no central confirmation available).

Baseline characteristics were generally well balanced, except for a higher proportion of patients in the ipatasertib plus paclitaxel arm with a disease-free interval of $>3$ years ( $40 \%$ vs $29 \%$ in the placebo plus paclitaxel arm) or a chemotherapy-free interval $>3$ years $(31 \%$ vs $24 \%$, respectively) (Table 1). Prior therapy was balanced between arms and included (neo)adjuvant chemotherapy in $55 \%$ of patients, endocrine therapy for aBC in $46 \%$, PI $3 \mathrm{~K} /$ mTOR inhibitor in $24 \%$ (predominantly everolimus) and CDK4/6 inhibitor in 26\%. According to European Society for Medical Oncology (ESMO) definitions [17], 18\% of patients had primary endocrine resistance and $45 \%$ had secondary endocrine resistance. A further $18 \%$ of patients did not meet the ESMO definitions for endocrine resistance but were deemed by the investigator to have visceral crisis. Within the subset of 120 patients who had received no prior endocrine therapy in the advanced setting, 18 (15\%) had primary endocrine resistance, 34 (28\%) had secondary endocrine resistance and $39(33 \%)$ had visceral crisis without endocrine resistance.

\section{Efficacy}

At the clinical cutoff date (January 17, 2020), the median duration of follow-up in the overall population was 12.9 months (range 0-23.3 months) and was similar in the two treatment arms. Median investigator-assessed PFS was 9.3 months in both arms $(95 \%$ confidence interval [CI] 8.0-11.0 months in the ipatasertib plus paclitaxel arm and 7.2-12.2 months in the placebo plus paclitaxel arm). The PFS hazard ratio was 1.00 (95\% CI 0.71-1.40; log-rank $p=0.997$ ) (Fig. 2a). The 1-year PFS rate was $38 \%$ (95\% CI 29-46\%) in the ipatasertib plus paclitaxel arm and $40 \%$ (95\% CI 29-52\%) in the placebo plus paclitaxel arm. IRC-assessed PFS results were consistent with investigator-assessed PFS (median 9.2 months [95\% CI 7.6-11.9 months] with ipatasertib plus paclitaxel vs 8.5 months [95\% CI 6.7-10.0 months] with placebo plus paclitaxel). The IRC-assessed PFS hazard ratio was 0.79 (95\% CI 0.56-1.13; Fig. 2b). Subgroup analyses of PFS showed consistent results across all populations analyzed 


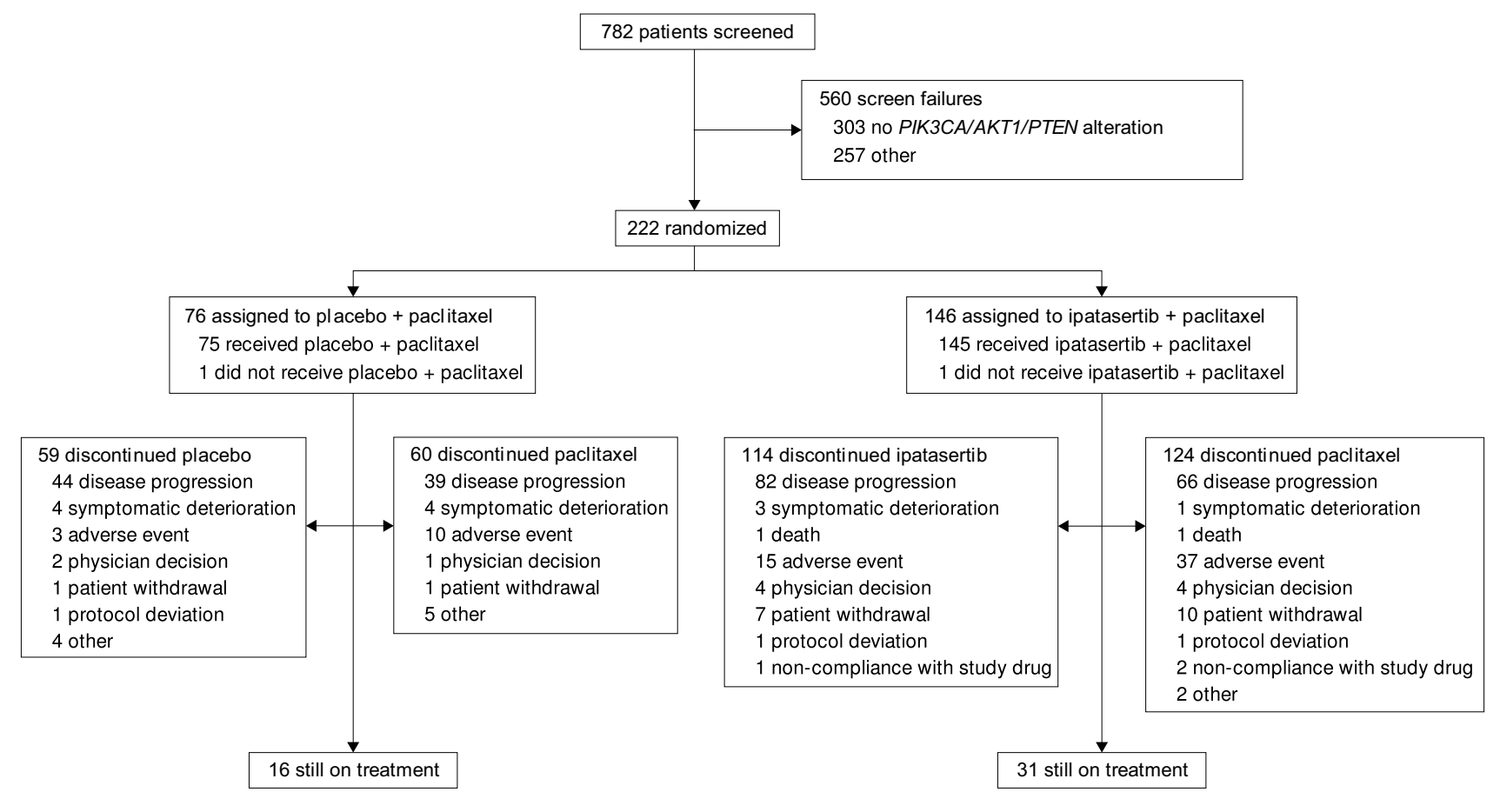

Fig. 1 Patient profile

and no subgroup deriving a benefit from ipatasertib was identified (Fig. 3).

Among the 144 patients in the ipatasertib plus paclitaxel arm and 75 in the placebo plus paclitaxel arm with measurable disease, the objective response rate was $47 \%$ in both arms (95\% CI 38-58\% and 35-59\%, respectively), including complete response in four patients $(3 \%)$ in the ipatasertib plus paclitaxel arm versus none in the placebo plus paclitaxel arm. The median duration of response was 9.2 months in both arms (95\% CI 7.2-11.3 months in the 67 responders in the ipatasertib plus paclitaxel arm; 95\% CI 6.8-12.5 months in the 35 responders in the placebo plus paclitaxel arm). The clinical benefit rate was 69\% (95\% CI $61-76 \%)$ in the ipatasertib plus paclitaxel arm and 65\% (95\% CI 53-76\%) in the placebo plus paclitaxel arm.

OS results were immature (deaths in $23 \%$ of the ipatasertib plus paclitaxel arm vs $29 \%$ of the placebo plus paclitaxel arm). At this interim analysis, median OS was not evaluable in ipatasertib-treated patients and 20.9 months (95\% CI 17.3-not evaluable) in the placebo plus paclitaxel arm (hazard ratio, $0.72,95 \%$ CI $0.42-1.24$ ).

\section{Patient-reported outcomes}

Completion rates for PRO questionnaires exceeded $80 \%$ in each arm up to cycle 23 and at the study drug discontinuation visit. Overall, 207 patients were evaluable for mean change from baseline in GHS/QoL (134 in the ipatasertib plus paclitaxel arm, 73 in the placebo plus paclitaxel arm).

Patients' GHS/QoL mean scores at baseline were 68.8 in the ipatasertib plus paclitaxel arm and 63.7 in the placebo plus paclitaxel arm, and were maintained in both treatment arms until Cycle 10 (at which point, less than half of the PRO-evaluable population in each arm remained on treatment, precluding meaningful analysis beyond Cycle 10) (Supplementary Figure S1). No clinically meaningful deterioration (i.e., $a \geq 10$-point decrease [18]) from baseline values was observed in either arm.

Median time to confirmed deterioration in pain (as measured by the pain scale of the EORTC QLQ-C30) was not evaluable in either treatment arm (confirmed deterioration in $38 \%$ of patients in the ipatasertib plus paclitaxel arm versus 30\% in the placebo plus paclitaxel arm). However, the Kaplan-Meier plot of time to confirmed $\geq 11$-point deterioration in pain from baseline showed a sustained separation of the curves at 6 months in favor of the placebo plus paclitaxel arm (hazard ratio, 1.36; 95\% CI 0.83-2.22) (Supplementary Figure S2).

\section{Safety}

At the clinical cutoff date, 31 patients (21\%) randomized to ipatasertib plus paclitaxel and $16(21 \%)$ randomized to placebo plus paclitaxel remained on treatment. Treatment 
Table 1 Baseline characteristics

\begin{tabular}{|c|c|c|}
\hline Characteristic & $\begin{array}{l}\text { Placebo }+ \text { paclitaxel } \\
(n=76)\end{array}$ & $\begin{array}{l}\text { Ipatasertib + paclitaxel } \\
(n=146)\end{array}$ \\
\hline Median age, years (range) & $56(28-77)$ & $57.5(30-81)$ \\
\hline Postmenopausal, $n(\%)$ & $59(78)$ & $113(78)^{\mathrm{a}}$ \\
\hline \multicolumn{3}{|l|}{ Region, $n(\%)^{\mathrm{b}}$} \\
\hline Asia-Pacific & $21(28)$ & $37(25)$ \\
\hline Europe & $36(47)$ & $74(51)$ \\
\hline North America & $6(8)$ & $7(5)$ \\
\hline Rest of world & $13(17)$ & $28(19)$ \\
\hline Prior (neo)adjuvant chemotherapy, $n(\%)^{\mathrm{b}}$ & $43(57)$ & $80(55)$ \\
\hline Prior PI3K/mTOR inhibitor, $n(\%)^{\mathrm{b}}$ & $17(22)$ & $36(25)$ \\
\hline Prior CDK4/6 inhibitor use, $n(\%)$ & $21(28)$ & $36(25)$ \\
\hline \multicolumn{3}{|l|}{ Disease-free interval, years, $n(\%)^{\mathrm{c}}$} \\
\hline$<1$ & $3(4)$ & $5(3)$ \\
\hline $1-3$ & $21(28)$ & $29(20)$ \\
\hline$>3$ & $22(29)$ & $58(40)$ \\
\hline No prior breast surgery & $23(30)$ & $43(29)$ \\
\hline Not available & $7(9)$ & $11(8)$ \\
\hline \multicolumn{3}{|l|}{ Chemotherapy-free interval, $n(\%)$} \\
\hline $1-3$ years & $18(24)$ & $29(20)$ \\
\hline$>3$ years & $18(24)$ & $45(31)$ \\
\hline No prior chemotherapy & $36(47)$ & $65(45)$ \\
\hline Not available & $4(5)$ & $7(5)$ \\
\hline Metastatic disease at baseline, $n(\%)$ & $74(97)$ & $141(97)$ \\
\hline \multicolumn{3}{|l|}{ Metastatic sites, $n(\%)^{\mathrm{d}}$} \\
\hline Lung & $35(46)$ & $52(36)$ \\
\hline Liver & $43(57)$ & $70(48)$ \\
\hline Bone & $54(71)$ & $95(65)$ \\
\hline Lymph node & $38(50)$ & $80(55)$ \\
\hline Visceral disease, $n(\%)$ & $63(83)$ & $114(78)$ \\
\hline \multicolumn{3}{|l|}{$\begin{array}{l}\text { No. of lines of prior endocrine treatment in } \\
\text { advanced setting, } n(\%)\end{array}$} \\
\hline 0 & $39(51)$ & $81(55)$ \\
\hline 1 & $17(22)$ & $34(23)$ \\
\hline 2 & $9(12)$ & $15(10)$ \\
\hline $3+$ & $11(14)$ & $16(11)$ \\
\hline \multicolumn{3}{|l|}{ Endocrine resistance status, $n(\%)^{\mathrm{e}}$} \\
\hline Primary & $14(18)$ & $26(18)$ \\
\hline Secondary & $32(42)$ & $67(46)$ \\
\hline Visceral crisis without endocrine resistance & $10(13)$ & $29(20)$ \\
\hline \multicolumn{3}{|l|}{ FMI status, $n(\%)^{\mathrm{f}}$} \\
\hline$P I K 3 C A / A K T 1$ alteration & $60(81)$ & $127(88)$ \\
\hline$P T E N$-only alteration & $14(19)$ & $17(12)$ \\
\hline
\end{tabular}

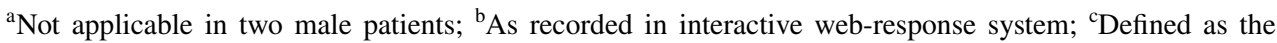
interval between final breast surgery with curative intent and initial diagnosis of locally advanced/metastatic breast cancer; ${ }^{\mathrm{d}}$ Missing in one patient in the placebo + paclitaxel arm; ${ }^{\mathrm{e}}$ Categories are mutually exclusive; ${ }^{\mathrm{f}} \mathrm{FMI}$ mutation status missing in two patients in each arm

$C D K$ cyclin-dependent kinase, FMI Foundation Medicine Inc, PI3K phosphoinositide 3-kinase

exposure is shown in Table 2. Mean paclitaxel dose intensity was similar in the two treatment arms, but the median duration of paclitaxel exposure was longer in the placebo plus paclitaxel arm than the ipatasertib plus paclitaxel arm. The most common AEs of any grade in patients who received ipatasertib plus paclitaxel were diarrhea (85\%; grade 3 in $12 \%$, grade 2 in $43 \%$, grade 1 in $30 \%$ ), alopecia (50\%) and nausea (41\%) (Table 3). Rash was slightly more 


\section{a}

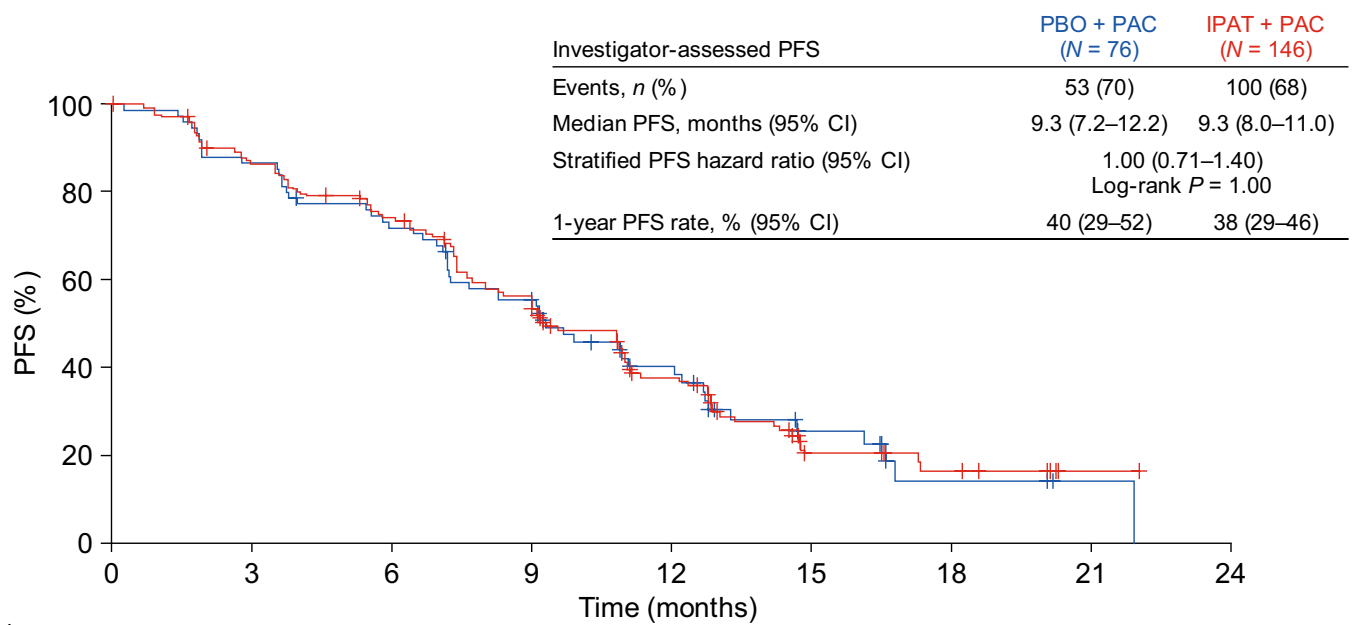

Number at risk $\mathrm{PBO}+\mathrm{PAC}$ IPAT + PAC

$\begin{array}{rrrr}76 & 65 & 53 & 40 \\ 146 & 121 & 102 & 75\end{array}$

$40 \quad 21$

21
40

9

$\begin{array}{ll}3 & 1 \\ 8 & 1\end{array}$

b

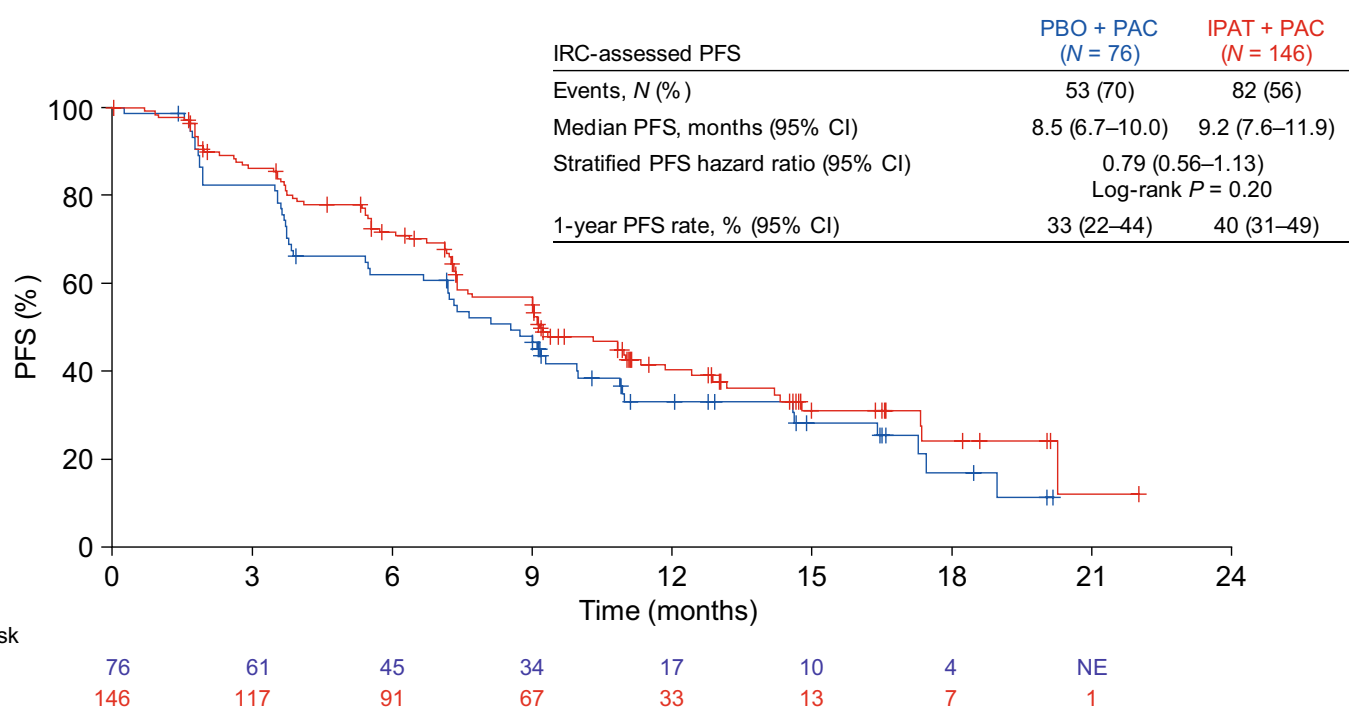

Number at risk $\mathrm{PBO}+\mathrm{PAC}$ IPAT + PAC

$\begin{array}{rrrr}76 & 61 & 45 & 34 \\ 146 & 117 & 91 & 67\end{array}$

Fig. 2 PFS. a investigator assessed; b IRC assessed. IPAT ipatasertib, $N E$ not evaluable, $P A C$ paclitaxel, $P B O$ placebo

common in ipatasertib-treated patients and the incidence of hyperglycemia was similar in the two arms.

Grade $\geq 3$ AEs occurred in 55\% of patients in the ipatasertib plus paclitaxel arm and $47 \%$ in the placebo plus paclitaxel arm. The most common grade $\geq 3$ AEs reported in either treatment group (ipatasertib plus paclitaxel vs placebo plus paclitaxel) were diarrhea (12\% vs $1 \%$, respectively [no grade 4 episodes]), neutrophil count decreased ( $9 \%$ vs $7 \%$ ), neutropenia ( $8 \%$ vs $9 \%$ ), peripheral neuropathy ( $7 \%$ vs $3 \%$ ), peripheral sensory neuropathy (3\% vs 5\%) and hypertension (1\% vs 5\%). Incidences of selected AEs of specific relevance to ipatasertib are shown in Supplementary Table S2. AEs leading to ipatasertib/placebo discontinuation included diarrhea (3\% vs $1 \%$ ), febrile neutropenia (1\% vs $0 \%$ ) and hyperglycemia ( $1 \%$ vs $0 \%$ ). The AEs most commonly leading to paclitaxel discontinuation were peripheral sensory neuropathy (5\% in both arms), peripheral neuropathy (6\% vs $1 \%$ ), neutrophil count decreased (1\% vs 3\%) and febrile neutropenia ( $2 \%$ vs $0 \%)$.

Most patients (92\% in the ipatasertib plus paclitaxel arm vs $82 \%$ in the placebo plus paclitaxel arm) received at least one dose of loperamide for diarrhea prophylaxis or treatment. The proportion receiving prophylactic loperamide was similar in the two treatment arms ( $61 \%$ vs $64 \%$, respectively) but a higher proportion of patients in the ipatasertib plus paclitaxel arm received loperamide to treat diarrhea (78\% vs $29 \%$, respectively). In the ipatasertib plus paclitaxel arm, $96 \%$ of diarrhea episodes ( 431 of 448 ) resolved. The median time to resolution of the first episode of diarrhea (any grade) was 15 days (95\% CI 8-18 days) and the median duration 


\begin{tabular}{|c|c|c|c|c|c|c|c|c|c|c|}
\hline \multirow[b]{2}{*}{ Baseline risk factors } & \multirow[b]{2}{*}{$\begin{array}{c}\text { Total } \\
n\end{array}$} & \multicolumn{2}{|c|}{$\begin{array}{c}\mathrm{PBO}+\mathrm{PAC} \\
(n=76)\end{array}$} & \multirow[b]{2}{*}{$\begin{array}{c}\text { Median } \\
\text { (months) }\end{array}$} & \multicolumn{2}{|c|}{$\begin{array}{c}\text { IPAT + PAC } \\
(n=146)\end{array}$} & \multirow[b]{2}{*}{$\begin{array}{l}\text { Median } \\
\text { (months) }\end{array}$} & \multirow[b]{2}{*}{ HR (95\% Wald Cl) } & \multirow[b]{2}{*}{$\begin{array}{c}\text { IPAT + PAC } \\
\text { better }\end{array}$} & \multirow[b]{2}{*}{$\begin{array}{c}\mathrm{PBO}+\mathrm{PAC} \\
\text { better }\end{array}$} \\
\hline & & $n$ & Events & & $n$ & Events & & & & \\
\hline $\begin{array}{l}\text { All patients } \\
\text { Age, years }\end{array}$ & 222 & 76 & 53 & 9.3 & 146 & 100 & 9.3 & $0.97(0.69-1.35)$ & & \\
\hline$<65$ & 170 & 63 & 44 & 9.2 & 107 & 73 & 9.1 & $0.96(0.66-1.39)$ & & \\
\hline$\geq 65$ & 52 & 13 & 9 & 9.9 & 39 & 27 & 11.0 & $0.99(0.46-2.11)$ & & \\
\hline \multicolumn{11}{|l|}{ Menopausal status at baseline } \\
\hline Premenopausal & 48 & 17 & 12 & 8.3 & 31 & 22 & 8.3 & $0.96(0.47-1.97)$ & & \\
\hline Postmenopausal & 172 & 59 & 41 & 9.9 & 113 & 76 & 9.6 & $0.99(0.68-1.45)$ & & \\
\hline \multicolumn{11}{|l|}{ Race } \\
\hline American Indian or Alaska Native & 7 & 3 & 3 & 9.2 & 4 & 1 & NE & $0.26(0.03-2.50)$ & & \\
\hline $\begin{array}{l}\text { Asian } \\
\text { Black or African American }\end{array}$ & 60 & 22 & 14 & 12.7 & 38 & 24 & 10.9 & $1.10(0.56-2.17)$ & & \\
\hline Black or African American & 5 & 3 & 3 & 7.7 & 2 & 1 & $\mathrm{NE}$ & $0.43(0.04-4.20)$ & & \\
\hline White & 138 & 45 & 32 & 8.3 & 93 & 68 & 7.7 & $0.97(0.64-1.49)$ & & \\
\hline \multicolumn{11}{|l|}{ Region (per eCRF) } \\
\hline Asia-Pacific & 57 & 21 & 13 & 12.7 & 36 & 23 & 10.9 & $1.05(0.52-2.11)$ & & \\
\hline Europe & 110 & 36 & 27 & 8.3 & 74 & 53 & 9.0 & $0.98(0.61-1.55)$ & & \\
\hline North America & 14 & 6 & 5 & 7.0 & 8 & 6 & 4.5 & $1.46(0.41-5.20)$ & & \\
\hline Rest of World & 41 & 13 & 8 & 9.9 & 28 & 18 & 12.2 & $0.84(0.35-2.00)$ & & \\
\hline \multicolumn{11}{|l|}{ ECOG performance status } \\
\hline 0 & 132 & 48 & 32 & 9.7 & 84 & 55 & 10.9 & $0.92(0.59-1.43)$ & & \\
\hline 1 & 90 & 28 & 21 & 9.2 & 62 & 45 & 7.4 & $0.98(0.58-1.65)$ & & \\
\hline \multicolumn{11}{|l|}{ Stage at initial diagnosis } \\
\hline Stage I-III & 162 & 54 & 37 & 9.1 & 108 & 79 & 8.4 & $1.14(0.77-1.69)$ & & \\
\hline Stage IV & 60 & 22 & 16 & 10.9 & 38 & 21 & 12.8 & $0.62(0.32-1.19)$ & $\mapsto$ & \\
\hline \multicolumn{11}{|l|}{ Disease-free interval } \\
\hline$<1$ year & 8 & 3 & 1 & $\mathrm{NE}$ & 5 & 3 & 7.3 & $0.87(0.09-8.51)$ & & \\
\hline $1-3$ years & 50 & 21 & 14 & 9.9 & 29 & 19 & 7.5 & $1.21(0.59-2.46)$ & & \\
\hline$>3$ years & 80 & 22 & 14 & 9.1 & 58 & 43 & 9.3 & $1.33(0.72-2.43)$ & & -1 \\
\hline No prior surgery & 66 & 23 & 18 & 10.9 & 43 & 26 & 11.0 & $0.72(0.39-1.31)$ & & \\
\hline Not available & 18 & 7 & 6 & 8.3 & 11 & 9 & 12.0 & $0.54(0.17-1.64)$ & $\longmapsto$ & \\
\hline \multicolumn{11}{|c|}{ Number of lines of prior endocrine therapy for advanced disease } \\
\hline 0 & 120 & 39 & 26 & 11.1 & 81 & 49 & 10.9 & $0.95(0.59-1.54)$ & & \\
\hline 1 & 51 & 17 & 12 & 10.9 & 34 & 25 & 9.0 & $1.03(0.51-2.06)$ & & \\
\hline 2 & 24 & 9 & 6 & 4.7 & 15 & 14 & 7.6 & $0.91(0.34-2.45)$ & $\vdash$ & -1 \\
\hline$\geq 3$ & 27 & 11 & 9 & 7.2 & 16 & 12 & 8.4 & $0.92(0.38-2.20)$ & $\vdash$ & \\
\hline \multicolumn{11}{|l|}{ Prior CDK4/6 inhibitor use } \\
\hline Yes & 57 & 21 & 15 & 5.6 & 36 & 26 & 7.3 & $0.85(0.45-1.60)$ & & \\
\hline No & 165 & 55 & 38 & 10.9 & 110 & 74 & 10.9 & $1.03(0.69-1.52)$ & & \\
\hline \multicolumn{11}{|l|}{ Prior PI3K/mTOR inhibitor use (per eCRF) } \\
\hline Yes & 34 & 12 & 9 & 9.1 & 22 & 17 & 8.4 & $1.26(0.55-2.92)$ & & -1 \\
\hline No & 188 & 64 & 44 & 9.7 & 124 & 83 & 10.8 & $0.94(0.65-1.36)$ & & \\
\hline \multicolumn{11}{|c|}{ Prior (neo)adjuvant chemotherapy (per eCRF) } \\
\hline Yes & 121 & 40 & 28 & 9.2 & 81 & 59 & 9.0 & $1.21(0.77-1.90)$ & & \\
\hline No & 101 & 36 & 25 & 9.7 & 65 & 41 & 11.0 & $0.74(0.45-1.23)$ & & \\
\hline \multicolumn{11}{|c|}{ Number of metastatic sites in patients with $\mathrm{mBC}$} \\
\hline $1-2$ & 84 & 27 & 15 & 12.7 & 57 & 36 & 11.0 & $1.27(0.69-2.33)$ & & \\
\hline$\geq 3$ & 130 & 46 & 36 & 7.5 & 84 & 61 & 8.4 & $0.85(0.56-1.28)$ & & \\
\hline Not applicable & 7 & 2 & 2 & 9.2 & 5 & 3 & 12.4 & $0.56(0.08-4.09)$ & & \\
\hline
\end{tabular}

Fig. 3 PFS in subgroups. Data for race 'multiple' (two patients in the placebo arm, none in the ipatasertib arm), race 'unknown' (one patient in the placebo arm without an event, nine patients in the ipatasertib arm) and number of metastatic sites in patients with $\mathrm{mBC}$ ' 0 ' (one patient in the placebo arm without an event) are not shown

of the first episode of grade $\geq 3$ diarrhea was 3 days $(95 \%$ CI 1-6 days).

Serious AEs were more common in the ipatasertib plus paclitaxel arm (19\%) than in the placebo plus paclitaxel arm (12\%). AEs were fatal in five patients (3\%) in the ipatasertib plus paclitaxel arm and one patient (1\%) in the placebo plus paclitaxel arm; two of these deaths were considered related to study treatment (grade 5 febrile neutropenia related to both drugs in the ipatasertib plus paclitaxel arm and grade 5 sepsis related to paclitaxel in the placebo plus paclitaxel arm; both patients had visceral crisis at screening). The remaining four deaths in the ipatasertib plus paclitaxel arm were from hospital-acquired pneumonia, respiratory distress, unexplained death, and general physical health deterioration/ road traffic accident (each reported in one patient). because hazard ratios could not be calculated with events in only one arm. ECOG Eastern Cooperative Oncology Group, eCRF electronic case report form, $H R$ hazard ratio, IPAT ipatasertib, $m B C$ metastatic breast cancer, $N E$ not evaluable, $P A C$ paclitaxel, $P B O$ placebo

\section{Discussion}

In Cohort B of the randomized phase 3 IPATunity 130 trial, adding ipatasertib to paclitaxel did not improve PFS in PIK3CA/AKT1/PTEN-altered HR+ HER2- aBC. Ipatasertib plus paclitaxel was well tolerated, and the safety profile of the regimen was consistent with the known risks of each agent. No new safety signals were identified. OS follow-up is ongoing.

The results from IPATunity 130 Cohort B are consistent with findings from the randomized, phase $2 \mathrm{BEECH}$ trial of the oral AKT inhibitor capivasertib in combination with first-line paclitaxel in HR+HER- aBC [19]. In BEECH, similar to the present trial, combining an AKT inhibitor with paclitaxel did not significantly improve PFS in either the overall population or the PIK3CA-altered population. The target patient population for IPATunity 130 Cohort B was patients with endocrine-resistant disease; however, only a quarter of patients had received prior CDK4/6 inhibitors. 
Table 2 Treatment exposure

Table 3 Summary of most common adverse events ( $\geq 20 \%$ any grade, $\geq 5 \%$ grade $\geq 3$ in either arm)

\begin{tabular}{lll}
\hline Treatment exposure & $\begin{array}{l}\text { Placebo + paclitaxel } \\
(n=75)\end{array}$ & $\begin{array}{l}\text { Ipatasertib+ paclitaxel } \\
(n=145)\end{array}$ \\
\hline Patients still on treatment, $n(\%)$ & $16(21)$ & $31(21)$ \\
Median (range) duration of treatment, months & $9.1(0-22)$ & $8.0(0-22)$ \\
Ipatasertib/placebo & $8.8(0-22)$ & $6.9(0-22)$ \\
Paclitaxel & & \\
Mean (SD) dose intensity, $(\%)^{\mathrm{a}}$ & $98.8(2.9)$ & $95.5(10.1)$ \\
Ipatasertib/placebo & $98.1(6.0)$ & $97.5(12.0)$ \\
Paclitaxel & & \\
Mean (SD) cumulative dose, mg & $\mathrm{NA}$ & $69691(48348)$ \\
Ipatasertib/placebo & $3727(2031)$ & $3313(2104)$ \\
Paclitaxel & $10(13)$ & $44(30)$ \\
Patients with AE leading to treatment discontinuation, $n(\%)$ & $16(11)$ \\
Ipatasertib/placebo & $3(4)$ & $38(26)$ \\
Paclitaxel & $10(13)$ & $67(46)$ \\
Patients with AE leading to dose reduction, $n(\%)$ & $20(27)$ & $50(34)$ \\
Ipatasertib/placebo & $6(8)$ & $38(26)$ \\
Paclitaxel & $18(24)$ & $86(59)$ \\
Patients with dose interruption, $n(\%)$ & $43(57)$ & $63(43)$ \\
Ipatasertib/placebo & $32(43)$ & $77(53)$ \\
Paclitaxel & $38(51)$ & \\
\hline
\end{tabular}

${ }^{a}$ With respect to total number of doses

$A E$ adverse event, $S D$ standard deviation

\begin{tabular}{|c|c|c|c|c|}
\hline \multirow[t]{2}{*}{ Adverse event, $n(\%)$} & \multicolumn{2}{|c|}{ Placebo + paclitaxel $(n=75)$} & \multicolumn{2}{|c|}{$\begin{array}{l}\text { Ipatasertib + paclitaxel } \\
(n=145)\end{array}$} \\
\hline & Any grade & Grade $\geq 3$ & Any grade & Grade $\geq 3$ \\
\hline Diarrhea & $28(37)$ & $1(1)$ & $123(85)$ & $17(12)$ \\
\hline Alopecia & $44(59)$ & 0 & $72(50)$ & 0 \\
\hline Nausea & $15(20)$ & 0 & $60(41)$ & $2(1)$ \\
\hline Neuropathy peripheral & $12(16)$ & $2(3)$ & $46(32)$ & $10(7)$ \\
\hline Anemia & $15(20)$ & 0 & $43(30)$ & $1(1)$ \\
\hline Vomiting & $5(7)$ & 0 & $42(29)$ & $3(2)$ \\
\hline Constipation & $23(31)$ & 0 & $39(27)$ & 0 \\
\hline Neutropenia & $18(24)$ & $7(9)$ & $38(26)$ & $12(8)$ \\
\hline Rash & $9(12)$ & 0 & $29(20)$ & $2(1)$ \\
\hline Fatigue & $18(24)$ & $3(4)$ & $27(19)$ & 0 \\
\hline Asthenia & $13(17)$ & $2(3)$ & $27(19)$ & $2(1)$ \\
\hline Peripheral sensory neuropathy & $22(29)$ & $4(5)$ & $23(16)$ & $4(3)$ \\
\hline Neutrophil count decreased & $17(23)$ & $5(7)$ & $23(16)$ & $13(9)$ \\
\hline Hyperglycemia & $9(12)$ & 0 & $20(14)$ & $3(2)$ \\
\hline ALT increased & $15(20)$ & $3(4)$ & $17(12)$ & $7(5)$ \\
\hline WBC decreased & $5(7)$ & $1(1)$ & $10(7)$ & $4(3)$ \\
\hline Lipase increased & $3(4)$ & $2(3)$ & $5(3)$ & $3(2)$ \\
\hline Hypertension & $4(5)$ & $4(5)$ & $7(5)$ & $2(1)$ \\
\hline
\end{tabular}

$A L T$ alanine aminotransferase; $W B C$ white blood cell 
Although it is tempting to hypothesize that enrollment of a less endocrine-resistant patient population could explain the lack of PFS benefit, subgroup analyses do not support this hypothesis. The subgroup of patients with greater exposure to prior endocrine therapy did not show enhanced benefit from ipatasertib (Fig. 3).

Another possible explanation for the lack of benefit is the higher proportion of patients discontinuing paclitaxel because of AEs in the ipatasertib arm, which may have compromised the efficacy of paclitaxel. Patients in the ipatasertib plus paclitaxel arm received a shorter duration and lower cumulative dose of paclitaxel than those in the placebo plus paclitaxel arm. This may have limited the ability to isolate the effect of ipatasertib. Of note, median PFS was identical in the two treatment arms and there was no signal of benefit from ipatasertib. There may be important lessons to learn from the duration and intensity of paclitaxel exposure, and the challenges of introducing a drug in HR+ HER2- breast cancer with side effects differing from those of endocrine therapies. Of note, a similar proportion of patients (approximately one-third) in each arm experienced a confirmed deterioration in pain, and patients' baseline quality of life was maintained while receiving ipatasertib plus paclitaxel treatment, showing no detrimental effect on patients' overall quality of life with ipatasertib.

Consistent with the safety profile of ipatasertib plus paclitaxel observed in the LOTUS trial [15], there was more allgrade diarrhea, nausea and vomiting with ipatasertib. The incidence of diarrhea was lower in IPATunity 130 Cohort B than in LOTUS, with only half as many ipatasertib-treated patients experiencing grade 3 diarrhea (11\% in IPATunity 130 Cohort B vs $23 \%$ in LOTUS). The observed reduction may be explained by the implementation of several diarrhea management measures in the IPATunity 130 trial design, including prophylactic loperamide administration, improved patient education and AE management guidance, as well as greater investigator awareness and familiarity with the drug.

Hyperglycemia has been observed in various clinical trials of drugs targeting the PI3K/AKT pathway [20-22] and is generally considered to be a class effect of these therapies. However, in IPATunity 130 Cohort B, the proportion of patients experiencing hyperglycemia was lower than in trials of other PI3K/AKT inhibitors [20, 23-25] and similar in the two treatment arms (14\% with ipatasertib plus paclitaxel vs $12 \%$ with placebo plus paclitaxel). The proportion of patients with grade $\geq 3$ hyperglycemia was low ( $2 \%$ vs $0 \%$, respectively).

Overall, results from IPATunity 130 Cohort B and the BEECH [19] trial differ from findings of trials combining a PI3K/AKT inhibitor with endocrine therapy (SOLAR-1 [20] and FAKTION [26]). PI3K/AKT signaling promotes estrogen-independent growth of HR+HER2- breast cancer cells, which can be inhibited by combining PI3K inhibitors with anti-estrogens $[8,92728,29]$. The SOLAR-1 randomized, phase 3 trial combined the PI3K inhibitor alpelisib with fulvestrant in patients with PIK3CA-mutant HR+ HER2- breast cancer [20] and the FAKTION trial combined the oral AKT inhibitor capivasertib with fulvestrant after relapse or progression on an aromatase inhibitor [26]. In line with preclinical findings, both of the trials showed a PFS benefit from the addition of a PI3K/AKT pathway inhibitor to endocrine therapy.

Considering all available data for AKT inhibition in HR+ HER2 $-a B C$, it appears that endocrine blockade may be essential for efficacy in this setting. AKT induces endocrine receptor signaling, which may counter the potential benefit of an AKT inhibitor. Taken together, these results suggest that the benefit of AKT inhibition will be greatest if estrogen receptors are targeted alongside AKT inhibition. Ongoing trials of ipatasertib in breast cancer focus on combinations with endocrine therapy and/or immunotherapy.

Supplementary Information The online version contains supplementary material available at https://doi.org/10.1007/s10549-021-06450-x.

Acknowledgements We thank the patients, their families, the investigators and study staff at participating sites, and members of the independent data monitoring committee. This work was supported by Genentech/Roche. Medical writing assistance was provided by Jennifer Kelly, MA (Medi-Kelsey Ltd), funded by F. Hoffmann-La Roche Ltd, Basel, Switzerland.

Authors' contributions NT, RD, JO'S, S-BK, SJI, CB, MJW, and MO were involved in the conception of the study. NT, RD, JO'S, S-BK, SJI, CB, SS, MJW, AM, and MO were involved in the methodology. NT, RD, JO'S, S-BK, SJI, CB, SS and MO were involved in the investigation. NT, RD, JO'S, S-BK, SJI, CB, SS, IB, ZN, and MO provided resources for the study. NT, RD, QL, S-JR, HH, MJW, AM and $\mathrm{MO}$ were involved in writing the first draft of the manuscript. QL was involved in the validation of the data and visualization. QL, MJW, and BK were involved in the formal analysis of the data. QL, S-JR, HH, MJW, BK, and AM were involved in the data curation. $\mathrm{S}$-JR and AM were involved in the supervision and administration of the project. All authors were involved in the review and editing of the final manuscript.

Funding This work was supported by Genentech/Roche. Medical writing assistance was provided by Jennifer Kelly, MA (Medi-Kelsey Ltd), funded by F. Hoffmann-La Roche Ltd, Basel, Switzerland.

Data availability Qualified researchers may request access to individual patient-level data through the clinical study data request platform (https://vivli.org/). Further details on Roche's criteria for eligible studies are available here (https://vivli.org/members/ourmembers/). For further details on Roche's Global Policy on the Sharing of Clinical Information and how to request access to related clinical study documents, see here (https://www.roche.com/research_and_development/ who_we_are_how_we_work/clinical_trials/our_commitment_to_data_ sharing.htm). 


\section{Declarations}

Conflict of interest N Turner has received honoraria for advisory/consultancy from AstraZeneca, Bristol-Myers Squibb, Lilly, Merck Sharpe and Dohme, Novartis, Pfizer, Roche/Genentech, Bicycle Therapeutics, Taiho, Zeno pharmaceuticals, Repare therapeutics; research grant/ funding (to institution) from AstraZeneca, BioRad, Pfizer, Roche/ Genentech, Clovis, Merck Sharpe and Dohme, Guardant Health. RA Dent has received honoraria from Roche, Novartis, Lilly, Pfizer, Eisai, Merck, AstraZeneca; travel/accommodation/expenses from AstraZeneca, Merck, Pfizer, Roche. J O'Shaughnessy has received honoraria for advisory/consultancy from AbbVie Inc., Agendia, Amgen Biotechnology, AstraZeneca, Bristol-Myers Squibb, Celgene Corporation, Eisai, Genentech, Genomic Health, GRAIL, Immunomedics, Heron Therapeutics, Ipsen Biopharmaceuticals, Jounce Therapeutics, Lilly, Merck, Myriad, Novartis, Odonate Therapeutics, Pfizer, Puma Biotechnology, Prime Oncology, Roche, Seattle Genetics, Syndax Pharmaceuticals, Takeda. S-B Kim has served as an advisor/consultant for Novartis, AstraZeneca, Lilly, Enzychem Lifesciences, Dae Hwa Pharmaceutical Co. Ltd, ISU Abxis, Daiichi-Sankyo; research grants/funding (to institution) from Novartis, Sanofi-Aventis, Kyowa-Kirin Inc, DongKook Pharm Co. SJ Isakoff has served as an advisor/consultant for Genentech, AbbVie, Hengrui, Immunomedics, Mylan, Puma, Oncopep Research; research grants/funding (to institution) from Genentech, AbbVie, Oncopep, AstraZeneca, Merck. C Barrios has received honoraria for advisory/consultancy roles from Boehringer Ingelheim, GSK, Novartis, Pfizer, Lilly, Roche/Genentech, Eisai, MSD, AstraZeneca, Bayer; research grants/funding (to institution) from AbbVie, Amgen, Astellas Pharma, AstraZeneca, Bristol-Myers Squibb, Celgene, Covance, Lilly, Medivation, Merck Serono, MSD, Novartis, Pfizer, PharmaMar, Roche/Genentech; travel/accommodation/expenses from Boehringer Ingelheim, GSK, Novartis, Pfizer, Lilly, Roche/Genentech, Eisai, MSD, AstraZeneca, Bayer. S Saji has received honoraria from Chugai, Eisai, AstraZeneca, Takeda, Novartis, Kyowa-Kirin, Eli Lilly, MSD, Pfizer; has served as an advisory/consultant for Chugai, Novartis, Kyowa-Kirin; research grants/funding (to institution) from Chugai, Eisai, Takeda, Novartis, Daiichi-Sankyo, Taiho Pharmaceutical. I Bondarenko reports no disclosures. Z Nowecki has received travel/accommodation/expenses from Roche. Q Lian is an employee of GNE/Roche and holds stocks/shares in Roche. S-J Reilly is an employee of Roche Products Ltd and holds shares in Roche. H Hinton is an employee of F. Hoffmann-La Roche Ltd and holds shares in Roche. MJ Wongchenko is an employee of Genentech and holds shares in Roche. B Kovic is an employee of Hoffmann-La Roche Limited. A Mani is an employee of Genentech and holds shares in Roche. M Oliveira has served as an advisor/consultant for GNE, GSK, PUMA Biotechnology, Roche, Seattle Genetics, Novartis; research grants/funding (to institution) from GNE, GSK, PUMA Biotechnology, Roche, Seattle Genetics, Novartis, Philips, AstraZeneca, Immunomedics, Boehringer Ingelheim, Zenith Epigenetics, Cascadian Therapeutics, Sanofi, Celldex, Bayer, Piqur; member of the executive board of the SOLTI Breast Cancer Research Group (non-remunerated).

Ethical approval The study protocol was approved by an institutional review board or independent ethics committee at each site. The trial was carried out in accordance with the principles of the International Conference on Harmonisation Guidelines for Good Clinical Practice, the Declaration of Helsinki, and all applicable national and local laws.

Consent to participate All patients provided written informed consent.

Consent for publication All patients participating in the trial provided written informed consent.
Open Access This article is licensed under a Creative Commons Attribution 4.0 International License, which permits use, sharing, adaptation, distribution and reproduction in any medium or format, as long as you give appropriate credit to the original author(s) and the source, provide a link to the Creative Commons licence, and indicate if changes were made. The images or other third party material in this article are included in the article's Creative Commons licence, unless indicated otherwise in a credit line to the material. If material is not included in the article's Creative Commons licence and your intended use is not permitted by statutory regulation or exceeds the permitted use, you will need to obtain permission directly from the copyright holder. To view a copy of this licence, visit http://creativecommons.org/licenses/by/4.0/.

\section{References}

1. Testa JR, Tsichlis PN (2005) AKT signaling in normal and malignant cells. Oncogene 24:7391-7393

2. Manning BD, Cantley LC (2007) AKT/PKB signaling: navigating downstream. Cell 129:1261-1274

3. Manning BD, Toker A (2017) AKT/PKB signaling: navigating the network. Cell 169:381-405

4. El Sayed R, El Jamal L, El Iskandarani S, Kort J, Abdel Salam M, Assi $\mathrm{H}$ et al (2019) Endocrine and targeted therapy for hormonereceptor-positive, HER2-negative advanced breast cancer: insights to sequencing treatment and overcoming resistance based on clinical trials. Front Oncol 9:510

5. Sobhani N, Roviello G, Corona SP, Scaltriti M, Ianza A, Bortul $\mathrm{M}$ et al (2018) The prognostic value of PI3K mutational status in breast cancer: a meta-analysis. J Cell Biochem 119:4287-4292

6. Mosele F, Stefanovska B, Lusque A, Tran Dien A, Garberis I, Droin N et al (2020) Outcome and molecular landscape of patients with PIK3CA-mutated metastatic breast cancer. Ann Oncol 31:377-386

7. Abraham J (2015) PI3K/AKT/mTOR pathway inhibitors: the ideal combination partners for breast cancer therapies? Expert Rev Anticancer Ther 15:51-68

8. Miller TW, Hennessy BT, González-Angulo AM, Fox EM, Mills GB, Chen $\mathrm{H}$ et al (2010) Hyperactivation of phosphatidylinositol-3 kinase promotes escape from hormone dependence in estrogen receptor-positive human breast cancer. J Clin Invest 120:2406-2413

9. Miller TW, Balko JM, Arteaga CL (2011) Phosphatidylinositol 3-kinase and antiestrogen resistance in breast cancer. J Clin Oncol 29:4452-4461

10. Lin J, Sampath D, Nannini MA, Lee BB, Degtyarev M, Oeh J et al (2013) Targeting activated Akt with GDC-0068, a novel selective Akt inhibitor that is efficacious in multiple tumor models. Clin Cancer Res 19:1760-1772

11. Blake JF, Xu R, Bencsik JR, Xiao D, Kallan NC, Schlachter S et al (2012) Discovery and preclinical pharmacology of a selective ATP-competitive Akt inhibitor (GDC-0068) for the treatment of human tumors. J Med Chem 55:8110-8127

12. Yan Y, Serra V, Prudkin L, Scaltriti M, Murli S, Rodríguez O et al (2013) Evaluation and clinical analyses of downstream targets of the Akt inhibitor GDC-0068. Clin Cancer Res 19:6976-6986

13. Saura C, Roda D, Roselló S, Oliveira M, Macarulla T, PérezFidalgo JA et al (2017) A first-in-human phase I study of the ATP-competitive AKT inhibitor ipatasertib demonstrates robust and safe targeting of AKT in patients with solid tumors. Cancer Discov 7:102-113

14. Isakoff SJ, Tabernero J, Molife LR, Soria J-C, Cervantes A, Vogelzang NJ et al (2020) Antitumor activity of ipatasertib combined 
with chemotherapy: results from a phase Ib study in solid tumors. Ann Oncol 31:626-633

15. Kim SB, Dent R, Im SA, Espié M, Blau S, Tan AR: LOTUS investigators, et al (2017) Ipatasertib plus paclitaxel versus placebo plus paclitaxel as first-line therapy for metastatic triple-negative breast cancer (LOTUS): a multicentre, randomised, double-blind, placebo-controlled, phase 2 trial. Lancet Oncol 18:1360-1372

16. Cocks K, King MT, Velikova G, de Castro G, Martyn St-James M, Fayers PM et al (2012) Evidence-based guidelines for interpreting change scores for the European Organisation for the Research and Treatment of Cancer Quality of Life Questionnaire Core 30. Eur J Cancer 48(11):1713-1721

17. Cardoso F, Senkus E, Costa A, Papadopoulos E, Aapro M, André $F$ et al (2018) 4th ESO-ESMO International Consensus Guidelines for Advanced Breast Cancer (ABC 4). Ann Oncol 29:1634-1657

18. Osoba D, Rodrigues G, Myles J, Zee B, Pater J (1998) Interpreting the significance of changes in health-related quality-of-life scores. J Clin Oncol 16(1):139-144

19. Turner NC, Alarcón E, Armstrong AC, Philco M, López Chuken YA, Sablin M-P et al (2019) BEECH: a dose-finding run-in followed by a randomised phase II study assessing the efficacy of AKT inhibitor capivasertib (AZD5363) combined with paclitaxel in patients with estrogen receptor-positive advanced or metastatic breast cancer, and in a PIK3CA mutant sub-population. Ann Oncol 30:774-780

20. André F, Ciruelos E, Rubovszky G, Campone M, Loibl S, Rugo HS et al; SOLAR-1 Study Group (2019) Alpelisib for PIK3CAmutated, hormone receptor-positive advanced breast cancer. N Engl J Med 380:1929-1940.

21. Busaidy NL, Farooki A, Dowlati A, Perentesis JP, Dancey JE, Doyle LA et al (2012) Management of metabolic effects associated with anticancer agents targeting the PI3K-Akt-mTOR pathway. J Clin Oncol 30:2919-2928

22. Curigliano G, Shah RR (2019) Safety and tolerability of phosphatidylinositol-3-kinase (PI3K) inhibitors in oncology. Drug Saf 42:247-262

23. Baselga J, Im SA, Iwata H, Cortés J, De Laurentiis M, Jiang Z et al (2017) Buparlisib plus fulvestrant versus placebo plus fulvestrant in postmenopausal, hormone receptor-positive, HER2-negative, advanced breast cancer (BELLE-2): a randomised, double-blind, placebo-controlled, phase 3 trial. Lancet Oncol 18:904-916

24. Di Leo A, Johnston S, Lee KS, Ciruelos E, Lønning PE, Janni W et al (2018) Buparlisib plus fulvestrant in postmenopausal women with hormone-receptor-positive, HER2-negative, advanced breast cancer progressing on or after mTOR inhibition (BELLE-3): a randomised, double-blind, placebo-controlled, phase 3 trial. Lancet Oncol 19:87-100

25. du Rusquec P, Blonz C, Frenel JS, Campone M (2020) Targeting the PI3K/Akt/mTOR pathway in estrogen-receptor positive HER2 negative advanced breast cancer. Ther Adv Med Oncol 12:1758835920940939

26. Jones RH, Casbard A, Carucci M, Cox C, Butler R, Alchami F et al (2020) Fulvestrant plus capivasertib versus placebo after relapse or progression on an aromatase inhibitor in metastatic, oestrogen receptor-positive breast cancer (FAKTION): a multicentre, randomised, controlled, phase 2 trial. Lancet Oncol 21:345-357

27. Miller TW, Balko JM, Fox EM, Ghazoui Z, Dunbier A, Anderson $\mathrm{H}$ et al (2011) ER $\alpha$-dependent E2F transcription can mediate resistance to estrogen deprivation in human breast cancer. Cancer Discov 1:338-351

28. Crowder RJ, Phommaly C, Tao Y, Hoog J, Luo J, Perou CM et al (2009) PIK3CA and PIK3CB inhibition produce synthetic lethality when combined with estrogen deprivation in estrogen receptorpositive breast cancer. Cancer Res 69:3955-3962

29. Bosch A, Li Z, Bergamaschi A, Ellis H, Toska E, Prat A et al (2015) PI3K inhibition results in enhanced estrogen receptor function and dependence in hormone receptor-positive breast cancer. Sci Transl Med. https://doi.org/10.1126/scitranslmed.aaa4442

Publisher's Note Springer Nature remains neutral with regard to jurisdictional claims in published maps and institutional affiliations.

\section{Authors and Affiliations}

\section{Nicholas Turner ${ }^{1,2}$ (1) . Rebecca A. Dent ${ }^{3}$ - Joyce O'Shaughnessy ${ }^{4}$. Sung-Bae Kim ${ }^{5}$. Steven J. Isakoff . Carlos Barrios $^{7}$. Shigehira Saji ${ }^{8} \cdot$ Igor Bondarenko $^{9} \cdot$ Zbigniew Nowecki $^{10}$. Qinshu Lian ${ }^{11} \cdot$ Sarah-Jayne Reilly ${ }^{12} \cdot$ Heather Hinton ${ }^{13}$. Matthew J. Wongchenko ${ }^{14} \cdot$ Bruno Kovic $^{15} \cdot$ Aruna Mani $^{16} \cdot$ Mafalda Oliveira $^{17}$}

1 Breast Unit, The Royal Marsden NHS Foundation Trust, Fulham Road, London SW3 6JJ, UK

2 Breast Cancer Now Research Centre, The Institute of Cancer Research, London, UK

3 Division of Medical Oncology, National Cancer Centre Singapore, Singapore, Singapore

4 Department of Medical Oncology, Texas Oncology, Baylor University Medical Center, US Oncology, Dallas, TX, USA

5 Department of Oncology, Asan Medical Center, University of Ulsan College of Medicine, Seoul, South Korea

6 Division of Hematology and Oncology, Massachusetts General Hospital, Boston, MA, USA

7 Latin American Cooperative Oncology Group, Oncology Research Service, Hospital São Lucas, PUCRS,

Porto Alegre, RS, Brazil
8 Department of Medical Oncology, Fukushima Medical University Hospital, Fukushima, Japan

9 Oncology and Medical Radiology Department, City Clinical Hospital No. 4, Dnipropetrovsk, Ukraine

10 Oncology Centre, Instytut im. Marii-Sklodowskiej, Warsaw, Poland

11 Biostatistics, Genentech, Inc, South San Francisco, CA, USA

12 Pharma Development, Roche Products Ltd, Welwyn Garden City, UK

13 Product Development Safety, F. Hoffmann-La Roche Ltd, Basel, Switzerland

14 Oncology Biomarker Development, Genentech, Inc, South San Francisco, CA, USA 
15 Patient-Centered Outcomes Research, Product Development, Hoffmann-La Roche Limited, Mississauga, ON, Canada

16 Product Development Oncology, Genentech, Inc, South San Francisco, CA, USA
17 Medical Oncology Department, Vall d'Hebron University Hospital, Vall d'Hebron Institute of Oncology (VHIO), Barcelona, Spain 